\title{
Objective blur assessment based on contraction errors of local contrast maps
}

\author{
David Boon Liang Bong • Bee Ee Khoo
}

Published online: 23 April 2014

(C) Springer Science+Business Media New York 2014

\begin{abstract}
Blur distortion appears in multimedia content due to acquisition, compression or transmission errors. In this paper, a method is proposed to predict blur severity based on the contraction errors of local contrast maps. The proposed method is developed from the observation that histogram distribution of natural image would contract according to the degree of blur distortion. In order to quantify the level of contraction, an efficient method of determining local contrast boundaries is used. The upper and lower bounds of local histogram distribution are defined for the original image, and outlying points beyond these bounds are used to form the local contrast map. For the corresponding patch of a blur image, the same values of upper and lower bounds are used and the local contrast map for the blur image could be produced. Total difference between local contrast maps of the original and blur images is the contraction errors which are used to derive the blur score. The proposed method has advantages in terms of computation efficiency, and is performed in the spatial domain without the need of data transformation, conversion or filtering. In addition, prior training is not required at all for the model. Implementation of the proposed method as a multimedia tool is useful for estimating blur severity in multimedia content. The performance of the proposed method is verified by using three different blur databases and compared to popular state-of-the-art methods. Experiment results show that the proposed blur metric has high correlation with human perception of blurriness.
\end{abstract}

Keywords Objective blur assessment · Contrast maps · Contraction errors · Histogram distribution · Spatial domain

\section{Introduction}

Blurriness is a common degradation for multimedia content such as multimedia messaging service (MMS) and web images. It is normally caused by imperfect image acquisition processes,

D. B. L. Bong $(\bowtie)$

Faculty of Engineering, Universiti Malaysia Sarawak, 94300 Kota Samarahan, Malaysia

e-mail: davidblbong@yahoo.com

D. B. L. Bong $\cdot$ B. E. Khoo

School of Electrical \& Electronics Engineering, Universiti Sains Malaysia, 14300 Penang, Malaysia

B. E. Khoo

e-mail: beekhoo@eng.usm.my 